\title{
New Certified Reference Materials for the determination of photometric accuracy in UV spectrophotometry
}

\author{
John P. Hammond C.Chem MRSC
}

Optiglass Ltd, 52/54 Fowler Road, Hainault, IG6 3UT, UK

\section{Introduction}

As you can see from the title, this article relates to the development of new Certified Reference Materials (CRMs), for use in determining the essential accuracy and linearity of the Transmission scale of a conventional $\mathrm{UV} / \mathrm{vis}$ spectrophotometer. In general conversation, (probably because as an instrumental technique, it's well over 50 years since the Beckman DU appeared on the market) we refer to these instruments as UV/vis spectrophotometers, and by inference consider them as one instrument. As we shall see later it's probably safest to say that there are two instruments under one cover. It is very easy to assume that the fundamental $X / Y$ instrumental scales of wavelength and Transmission will remain linear and "under control" across the whole range of values, if validation measurements are performed in one region of the control space. As we shall see later, this is a very dangerous assumption to make. If the system is to be used in the UV region (190-350 $\mathrm{nm}$ ) then at least the wavelength and photometric accuracy must be determined in this region, using appropriate reference materials (UV CRMs).

This article is structured as follows.

First, we discuss the key topic of Traceability. Without this essential requirement defined and considered as the primary requirement, any reference material developments are meaningless, and the certification produced there after, is not really worth the paper that it is written on.

Then we move on to discuss the Operational Qualification (OQ) of the instrument, to establish the essential "evidence of control" in your laboratory. In this section we briefly discuss the use of Neutral Density glass filters, as these materials do embody all the essential characteristics required of a CRM for the OQ of a visible spectrophotometer.

Following on from this section we move on to discuss the UV region of the spectrum, and the use of a holistic approach to establish the "evidence of control" in this region, using potassium dichromate solutions. As we shall see, by definition this procedure establishes a Performance Qualification (PQ) of the laboratory, so we are missing that essential Operational Qualification in the UV region?

So what UV CRMs are current available-unfortunately, very few. We need new materials, or materials that isolate the preparation and use of available solution references, from the actual measurement, and one such CRM is described below.

\section{Traceability:}

\section{an essential}

\section{requirement}

Traceability means different things to different people. If we look at the ISO International Vocabulary of Basic and General Terms in Metrology, ISO, 1993 definition.

"Traceability is the property of the result of a measurement or the value of a standard whereby it can be related to stated references, usually national or international standards, through an unbroken chain of comparisons all having stated uncertainties."

But this has to be broadening into what this requirement means in an international marketplace. An excellent summary document is the Co-operation on International Traceability in Analytical Chemistry (CITAC) paper on "Traceability in Chemical Measurement". This positioning paper clearly described the role that traceability has to play in a global marketplace, where ISO 17025 accreditation has the anchor role to play as the Quality system provider. ${ }^{1}$

As we shall see shortly, ISO 17025 has "come of age" as the standard that provides the international aspect to any laboratory measurement process, since it's conversion last year from ISO Guide 25. In all aspects, it provides the control framework to assist the production of comparable measurements. The role of ISO 17025 and the associated ISO Guides in establishing Mutual Recognition Agreements, in Europe under the European Co-operation on Accreditation (EA) umbrella, and on the Asian-Pacific Rim, within the Asian Pacific Laboratory Accreditation Co-operation (APLAC) is clearly explained on these organisations websites. ${ }^{2.3}$ Now under the International Laboratory Accreditation Co-operation (ILAC) agreement we have a truly international base.

This extract below is taken directly from the ILAC web site at www.ilac.org. As you can see we are now at the start of the future.

\section{ILAC Neus - 2 Nov 00}

Signing of International Arrangement to Enhance Trade

An international arrangement, signed.in Washington, DC, on 2 November 2000, will enhance the acceptance of technical data accompanying goods crossing national borders. The Arrangement, which involves 37 member bodies from 28 economies represented at the General Assembly of the International Laboratory Accreditation Cooperation (ILAC), means that goods tested in one country by a laboratory that is accredited under a signatory to the Arrangement, 
will be accepted by other signatories. This is a major step towards reducing or eliminating the need for re-testing of the goods by the importing country.

The Arrangement enters into force from 31 January 2001

Belinda Collins, Chair of ILAC, noted the significance of the signing, "For many years, the retesting of goods by an importing country has been considered as a major technical barrier to trade. The World Trade Organization (WTO) identified such technical barriers as a major concern to world trade since the mid-1970s. Such barriers can not only add significant cost to goods entering a country, but can also delay, and in some cases prevent, the goods being accepted by foreign markets."

Dr. Collins further explained that "ILAC has been working towards overcoming these technical barriers for the last two decades by encouraging the development of regional recognition arrangements culminating in today's global recognition arrangement among representative bodies in each country. This will facilitate the acceptance of goods already tested by an accredited laboratory. Thus, goods tested in one country should enjoy easier access to foreign markets participating in the Arrangement."

The key to the Arrangement is the developing network of accredited testing and calibration facilities around the globe that are evaluated and recognized as being competent by specific authorities, known as laboratory accreditation bodies. These bodies are located in many economies and many of them participate in ILAC.

The following economies will participate in the Arrangement:

Australia, Belgium, Brazil, Canada, People's Republic of China, Czech Republic, Denmark, Finland, France, Germany, Hong Kong, China, India, Ireland, Italy, Japan, Republic of Korea, The Netherlands, New Zealand, Nonway, Singapore, South Africa, Spain, Sweden, Switzerland, Chinese Taipei, United Kingdom, United States of America, Vietnam.

A cornerstone of the new Arrangement is the utilization of existing or developing regional arrangements established in the Americas, the Asia Pacific region, Europe and Southern Africa. The bodies participating in these regional arrangements are responsible for maintaining the necessary confidence in accreditation bodies from their region that are signatories to the new ILAC Arrangement.

Mike Peet, Chair of the ILAC committee that developed the new Arrangement, explained the basis for the Arrangement's implementation by the international community: "Now that the Arrangement is in place, the next crucial step is for governments to take advantage of this Arrangement by using it to further develop or enhance trade agreements."
"There is now a firm foundation in place for manufacturers and exporters that have their goods tested by accredited laboratories to enjoy greater market access, less costs associated with re-testing, and overall greater competitiveness in global markets", he explained.

Established in 1977, ILAC is the peak international forum for the harmonization of laboratory accreditation procedures as a means of reducing technical barriers to trade, and the promotion of laboratory accreditation as a mechanism to enhance confidence in testing and calibration facilities, both domestically and internationally.

A truly global representation of what international agreement means.

So now having established the importance of international traceability, and the important part that ISO 17025 has to play in all aspects of that requirement, what is a "stated and/or internationally agreed suitable CRM"?

First, it can be a primary material from a recognised national standards laboratory. For example, the National Physical Laboratory (NPL), UK, and the National Institute of Standards and Technology (NIST), USA, just two of these reference laboratories located around the globe. However, very often the "National Laboratories" cannot meet the demand (now international) for appropriate reference materials, and one logical step is for these organisations to accredit secondary producers via an ISO 17025 certification process. This is the fundamental requirement that has driven NIST to introduce the NIST Traceable Reference Material (NTRM) programme for Neutral Density glass filters. In the UK, whilst there is currently no equivalent of the NTRM programme, producers of secondary reference materials can establish a similar traceability "chain" by obtaining ISO 17025 accreditation from the United Kingdom Accreditation Service (UKAS).

\section{OQ of photo-}

\section{metric accuracy in the UV region}

The surprising lack of UV CRMs for such an established technique has meant that up to now two alternative approaches have had to be used for the determination of photometric accuracy. Either, visible range CRMs are used and the qualification extrapolated into the UV region, or a holistic approach is taken where UV reference solutions are prepared in the laboratory to specified requirements, and the competence of the whole environment to generate quality data is established. As one will see from the procedure defined below, either of these two routes suffers from serious limitations, which would be eliminated by the availability of suitable UV CRMs.

\section{Visible neutral density filters}

All current UV/vis instrument manufacturers will at least specify the Transmission scale performance of their instruments in terms of the measured Absorbance of Neutral Density filters, usually at $546 \mathrm{~nm}$.

As we can see, here are some of the advantages of these Neutral Density glass filters.

- Available for over 20 years as primary Certified Reference Materials from the National Physical Laboratory (NPL), UK, and the National Institute of Standards and Technology (NIST), USA - Easily handled

- Low temperature coefficient

- Homogeneous

- Now available meeting CITAC traceability requirements as a NTRM

However, it we already have already stated its dangerous to assume that a spectrophotometers transmission characteristics in the UV and visible regions are matched.

The potential difference in energy levels between the UV and visible regions can be likened to the change experienced by a river, as shown in the photograph in Figure 1.

Some of the more enlightened manufacturers will also quote specifications in compliance with European Pharmacopoeia requirements, which by design must comply with the measurement of potassium dichromate solutions in the $60 \mathrm{mg} \mathrm{L}^{-1}$ region.

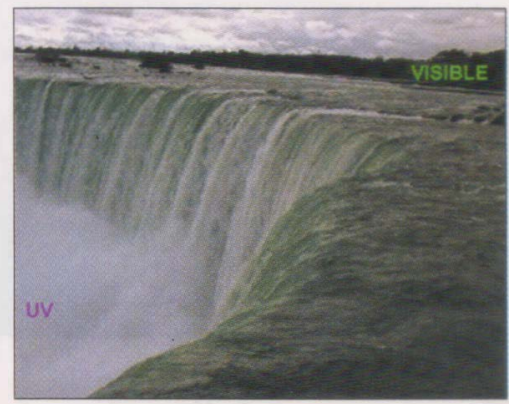

Figure 1. The Niagara river at Horseshoe Falls, Canada. 


\section{Use of potassium dichromate solutions}

So lets look at how the UV region is approached currently, if at all. Either a $57-63 \mathrm{mg} \mathrm{L}^{-1}$ solution of potassium dichromate in sulphuric acid is prepared according to the European Pharmacopoeia, or NIST SRM 935a is used to prepare a linear set of concentration solutions at $20,40,60$ and $80 \mathrm{mg} \mathrm{L}^{-1}$ levels in perchloric acid.

I use the additional comment "if at all" because it is the very fact that this is a holistic process, testing the whole laboratory. This does place this procedure squarely in the Performance Qualification (PQ) area of any Qualification plan. Whilst an extremely valuable source of information to a laboratory, it is very often substituted by a more appropriate $\mathrm{PQ}$ using the analyte to be routinely measured, and the $\mathrm{OQ}$ procedure in the UV region omitted. This is fine if the results of the PQ fall within expected parameters, but what if they don't, where does the error lie? The laboratory usually resorts back to preparing the appropriate potassium dichromate solutions, but as already has been stated, this is also a $P Q$, where many factors affect the result, not just the $O Q$ of the instrument. Therefore, the quality of the control of the procedure cannot be isolated from the quality of the instrument measurement.

One such control factor is cleanliness, because of the natural oxidising capability of the acidic dichromate solution. If you look at the basic reaction of the simple "Breathalyser test", and perform the appropriate calculations. Less than $0.04 \mu \mathrm{L}$ of ethanol effectively reduces completely the $60 \mathrm{mg} \mathrm{L}^{-1}$ potassium dichromate solution contained in a standard spectrophotometer cell.

\section{Options for UV OQ}

In practice, you have the limited availability of the primary Metal on Quartz filters; available from the example National Laboratories already quoted. These filters are expensive, and by their very nature work using reflection rather than absorption to achieve varying transmission levels. This may cause problems with some instrumental optical designs, when you consider that at the $2.0 \mathrm{~A}$ level, $99 \%$ of the incident beam is being reflected back down the optical path? Also, historically, these

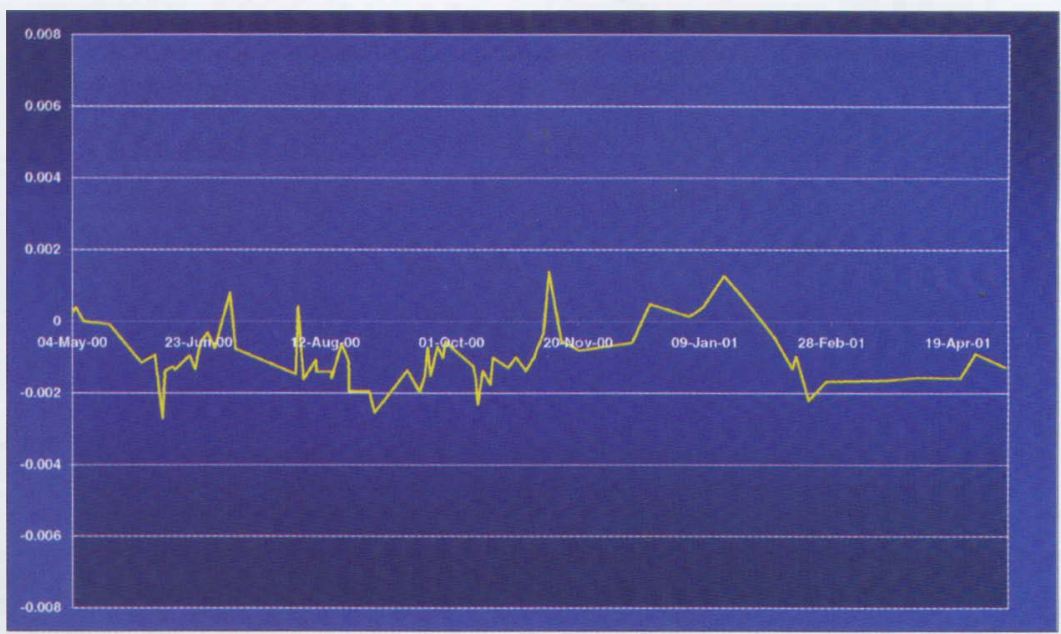

Figure 2. Variation of a $20 \mathrm{mg} \mathrm{L}^{-1}$ potassium dichromate reference over a period of 12 months.

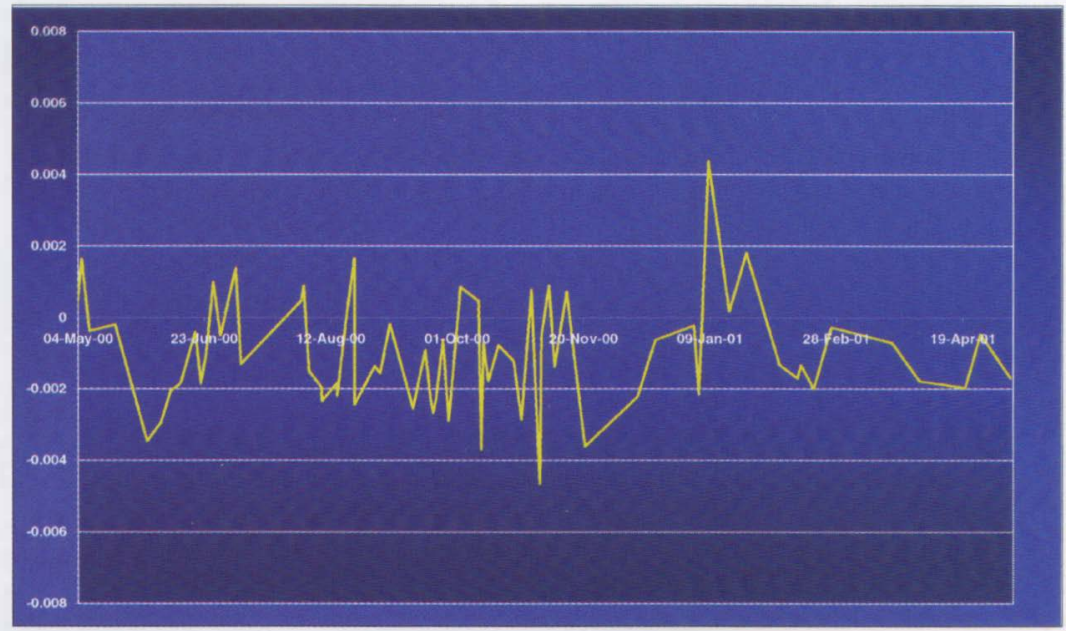

Figure 3. Variation of a $100 \mathrm{mg} \mathrm{L}^{-1}$ potassium dichromate reference over a period of 12 months.

materials are not as homogeneous as the Neutral Density glass filters.

For the reasons already discussed, if you split the potassium dichromate preparation process from the actual measurement process then these solutions can be used to $O Q$ the instrument.

This can be achieved essentially by sealing the solution in either ampoules or spectrophotometer cells. However, ampouled solutions still need to be transferred quantitatively to clean, unscratched, cells and again isolation of "control" from "measurement" becomes impossible? Therefore considering sealed cells as the only alternative, once again the traceability requirements must be emphasised, and the role that ISO 17025 can again play in the process cannot be overstated.

An example of such materials is the Starna range of RM sealed Dichromate materials from Optiglass. These UV
CRMs are prepared from the solid NIST SRM 935a Potassium Dichromate, in accordance with NIST Special Publication SP260-54. One essential physical characteristic required of a reference material is good stability with time. Figures 2 and 3 show the variation of a 20 and $100 \mathrm{mg} \mathrm{L}^{-1}$ potassium dichromate reference about a mean value over a 12 month period.

In conclusion, this research has shown that under the right conditions, potassium dichromate solutions sealed in cells are sufficiently stable, and have all the prerequisites to be calibrated as an UV Certified Reference Material.

\section{References}

1. www.fi/ket/citac/traceability.pdf. 2. www.european-accreditation.org. 3. www.ianz.govt.nz/aplac. 